\title{
Keeping up with YOUR Scientific Journals and YOUR Challenges
}

\author{
Barbara Catt, IPAC Canada President
}

In the summer edition of the Canadian Journal of Infection Control, Victoria Williams and Devon Metcalf referenced the Wellcome Trust and the impact of publications during the COVID-19 pandemic. As a segue into their article, and with the arrival of a new infection such as COVID-19, it has left many of us Infection Prevention and Control (IPAC) professionals trying to keep up with the latest evidence as we struggle to be grounded in the science at the same time due to the volumes of research to review. In a statement by the Wellcome Trust in January 2020, they expressed the necessity for making any information available that might have value in combatting a crisis, including research findings and data relevant to the COVID-19 pandemic. In the context of a public emergency of international concern, this data is important as it informs the public health response and helps to save lives. We need to see the evidence and findings quickly in order to assist with important decisions, guidance, and recommendations.

Similarly, the World Health Organization (WHO) has developed a comprehensive database for scientific findings and knowledge on COVID-19. This WHO COVID-19 database is updated weekly and includes searches of bibliographic databases, hand searching, and the addition of other expertreferred scientific articles. As well, the $\mathrm{WHO}$ database represents a comprehensive multilingual source of current literature on the COVID-19 topic. For more information on this, please access: https:// www.who.int/emergencies/diseases/novel-coronavirus-2019/globalresearch-on-novel-coronavirus-2019-ncov.

Many IPAC Professionals will use their ministry guidance documents to assist in some of the IPAC decisions within their provinces and territories. However, some of those documents are not always clear and can be interpreted differently. In these cases, it is important to review the literature. But it is also good to note that there may not be a publication to support practice, and therefore you may need to rely on experiences. This may be experiences from our colleagues who have had first-hand involvement with some of those questionable practices. Some of you may or may not recall the reprocessing of N95 respirators in March 2020 when demands left very little supply. This is an example of stepping outside of the comfort zone for IPAC professionals and recognizing that reprocessing of N95 respirators needed to be done at that time.

It is clear that we need to network with our IPAC colleagues such as the local IPAC chapters. I am hoping that during this time, IPAC professionals will draw from one another and share their experiences of what worked well and even those experiences that did not go so well. We are in this together and as a team, the IPAC Canada Team, we will get through this! Stay safe and well.

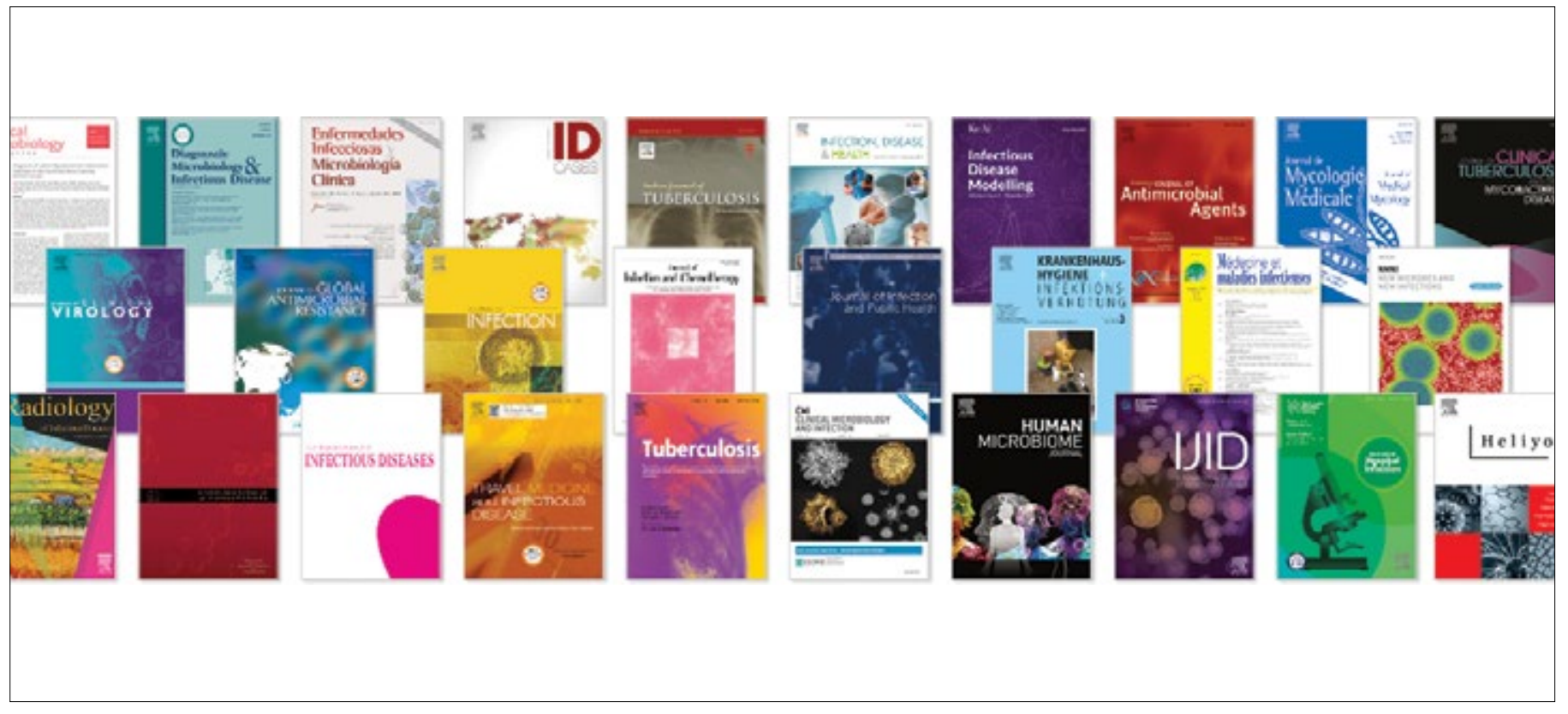

https://doi.org/10.36584/CJIC.2020.010 